This is a self-archived version of an original article. This version may differ from the original in pagination and typographic details.

Author(s): Jalasvuori, Matti

Title: Silent Rain : Does the atmosphere-mediated connectivity between microbiomes influence bacterial evolutionary rates?

Year: 2020

Version: Accepted version (Final draft)

Copyright: (c) 2020 FEMS

Rights: In Copyright

Rights url: http://rightsstatements.org/page/InC/1.0/?language=en

Please cite the original version:

Jalasvuori, M. (2020). Silent Rain : Does the atmosphere-mediated connectivity between microbiomes influence bacterial evolutionary rates?. Fems Microbiology Ecology, 96(7), Article fiaa096. https://doi.org/10.1093/femsec/fiaa096 


\title{
Silent Rain: Does the atmosphere-mediated connectivity between microbiomes influence bacterial evolutionary rates?
}

Matti Jalasvuori

University of Jyvaskyla, Department of Biological and Environmental Science, Nanoscience Center, Jyvaskyla, Finland

\begin{abstract}
Air carries a vast number of bacteria and viruses over great distances all the time. This leads into the continuous introduction of foreign genetic material to local established microbial communities. In this perspective, I ask whether this silent rain may have a slowing effect on the overall evolutionary rates in the microbial biosphere. Arguably, the greater the genetic divergence between gene "donors" and "recipients", the greater the chance that the gene product has a deleterious epistatic interaction with other gene products in its genetic environment. This is due to the long-term absence of check for mutual compatibility. As such, if an organism is extensively different from other bacteria, genetic innovations are less probable to fit to the genome. Here genetic innovation would be anything that elevates the fitness of the gene vehicle (e.g. bacterium) over its contemporaries. Adopted innovations increase the fitness of the compatible genome over incompatible ones, thus, possibly tempering the pace at which mutations accumulate in existing genomes over generations. I further discuss the transfer of bacteriophages through atmosphere and potential effects that this may have on local dynamics and perhaps phage survival.
\end{abstract}

Keywords: bacterial evolution, bacteriophages, atmosphere, evolutionary rate, genetic compatibility, genetic innovations 


\section{Foreword}

Bacteria and Archaea are present almost everywhere on our planet, vastly outnumbering other life forms (for simplicity, term bacteria hereafter refers to both domains of life). In this microbial biosphere, each individual cell contains genetic material that is subjected to selection via various environmental processes. In the traditional Darwinian view, mutations bring about new genetic variants for selection to act on, and it is these mutations that more or less dictate the pace at which the genomes, or in case of bacteria, probably the pangenomes, change over time. Nowadays we also acknowledge horizontal gene transfer as a notable source of (beneficial) genetic changes in bacteria (i.e. the access to the pangenome). These two modes of evolution are not mutually exclusive and occur simultaneously. In this perspective, I set to explore the possibility that these modes may be in direct "competition" against each other and speculate on the notion that intrinsic qualities of our planet may be sometimes tipping the scale in the favor of one of them.

Bacteria are known to interact and compete with their contemporaries, although initial competition may later evolve into stable co-existence due to e.g. niche partitioning (Ghoul and Mitri, 2016). Bacteria share (although often unwillingly) goods and exchange genes with each other (Morris et al., 2012; Jalasvuori and Koonin, 2015; Morris, 2015). Other entities such as bacteriophages, unicellular eukaryotes, plants and animals contribute to these interaction networks (McFall-Ngai et al., 2013; Cairns et al., 2018; Hiltunen et al., 2018). While the details in the complexity of microbial interactome are difficult to decipher, general trends can be at least recognized and sometimes mechanistically understood (Jalasvuori, 2012; Corel et al., 2016; Bonachela et al., 2017). I am lured to draw a crude analogy to the kinetic theory of gas: the dynamics of individual molecules (or here bacteria) are almost impossible to know, yet the behavior of the collective can be described with (some) accuracy. For example, we can identify overall speciation and extinction rates for bacteria (Stilianos et al., 2018) while we cannot accurately predict how many mutations any particular strain accumulates when facing, for example, new stressors (Cairns et al., 2016).

\section{High Skies of Life}

Atmosphere is now known to be full of bacteria and viruses (DeLeon-Rodriguez et al., 2013). The skies are not only a notable player in global biogeography, i.e. distribution species and ecosystems in geographical space and time, but it can be considered as a habitat of its own (Womack et al., 2010). Around $10^{7}$ bacterial cells and $10^{9}$ viruses are deposited from above the atmospheric boundary level (which means they come from afar) to each square meter every single day (Reche et al., 2018). While this number is small compared to the amount of existing bacteria and viruses in the area where they land, the continuous rain of "alien" 
(i.e. distantly originating) microbes may still be modulating the local interactions enough to provide significant evolutionary effects. Although, it must be noted that, this interaction may become notable only when it is observed at a sufficiently large temporary scales. Naturally, there are numerous other means by which bacteria traverse space between ecosystems but none of them are likely to be as constant and prevalent when compared to continuous aerosolization and windway carriage. The composition of the microbial fallout is also changing over time, at least within areas affected by Saharan dust outbreaks, but there can be interannual similarity (Barberán et al., 2014). Therefore, also the genes that fall are likely to have seasonal variation, yet annual resemblance. Nevertheless, I here discuss a few possible ways by which the atmosphere and its "genetic content" may have an impact on overall bacterial evolution. In other words, there may be evolutionary trends that exist just because of the atmosphere-mediated global genetic connections across habitats. These trends are inherent part of our ecosystem and thus difficult to isolate and analyze deterministically. As such, you may wish to approach this paper as a hypothesis, speculation or just a thought exercise for evaluating whether we genuinely understand the grand schemes behind the evolution of prokaryotes.

\section{Viral fallout: Red Queen to rule them all?}

Arms race between hosts and parasites is well-known for various types of organisms and suggested to be one of the key players in evolutionary change. The race between bacteria and their viruses is one of the best studied, at least on a molecular level (Labrie et al., 2010; Betts et al., 2018). The presence of phages often select for bacteria that have in one way or another lost the phage exploited receptors, hence making phages unable to infect the cell. Phages can also induce hosts to mask their receptors and elevate mutation rates. On the other hand, also opposite effects have been observed. Here, phages decrease the selection for mutators by making the evolution of social cheats less favorable ( $O^{\prime}$ Brien et al., 2013). Yet, defensive tools against phages are many. CRISPR and restriction modification systems of bacteria are also relatively well-studied defensive mechanisms that have developed in response to phages and can have long-term arms race cycles in natural habitats (Laanto et al., 2017). Certain bacteria may also secrete molecules that disrupt receptor binding (Kronheim et al., 2018) or they can commit suicide before new phage particles have time to assemble within the host cell (Labrie et al., 2010). In more general terms it is obvious that the arms race between bacteria and their viruses has played a significant role in bacterial evolution.

In laboratory conditions, Red Queen evolution between a virus and its bacterial host has been demonstrated repeatedly (Brockhurst et al., 2007; Koskella and Brockhurst, 2014; Betts et al., 2018). Phages cause bacteria to diverge more rapidly, and future bacteria are often resistant to previous versions of the virus. Correspondingly, phages co-evolve, adapting to infect the phage resistant mutants. Phages are 
also said to drive bacterial evolution. They are selecting against competitively dominant mutants and thus allowing more variability to thrive within bacterial communities (Rodriguez-Valera et al., 2009). Yet, do we know where phages are "driving" bacteria, in evolutionary terms, if anywhere?

Phages are very host specific, which states that a certain phage usually infects only some strains of a particular bacterial species. Meta-analysis by Flores and colleagues indicated that infection patterns are nested, in other words, hard to infect bacterial hosts are infected by generalist phages, not by specialists (Flores et al., 2013). Altogether, given the observed host specificity of phages and the Red Queen evolutionary arms race between phages and bacteria, one could speculate that in any particular environment it is mostly the co-evolved phages that are able to infect bacteria - the ones that were running alongside the Red Queen. However, experimental evolution has demonstrated that this is not necessarily the case. Migration of phages to locally adapted host-phage communities actually provided the parasite (phages) an evolutionary advantage over phages that evolved only locally (Morgan et al., 2005). Evolutionary experiments conducted as a part of soil community (in comparison to controlled cultures grown in high nutrient broth) also suggested that bacteria do evolve but may not co-evolve with their phages (Gomez and Buckling, 2011). The cost of phage-resistance may become relevant only in low nutrient environment such as in soil and hence bacterial population may be able to sustain resistance against only the co-occurring phages and not foreign phages. Now, if atmosphere carries "foreign" phages across bacterial habitats all the time, and if the phages actually find hosts in their new environment, this migration is likely to play a role in the evolutionary dynamics of local bacteria. Indeed, Braga and colleagues have suggested that the host-phage pairs could be seen as an evolutionary unit (holobiont) that is in fact the organism that speciates into particular ecological conditions (Braga et al., 2018). Phage fallout may continuously introduce new holobionts for selection to act on.

Phages are continuously being isolated for bacteria that emerged from distant locations. This is true, among others, for phage therapy trials where phages against antibiotic resistant gram-negative Enterobactericeae are found all over the world (Örmälä and Jalasvuori, 2013; Mattila et al., 2015). Same applies for marine and, apparently to, fresh water bacteria (Wolf et al., 2003; Laanto et al., 2011; Breitbarb et al., 2018). Notably, Atanasova and colleagues showed that infective viruses against halophilic bacteria and archaea are sometimes present all over the planet despite of the hosts being niche specialists and having very fragmented distribution of possible habitats (Atanasova et al., 2012). Separation in time is not a limiting factor either. New phages can be readily isolated for bacteria that have remained in culture collections for a century (Örmälä and Jalasvuori, 2013). The other way around, i.e. whether hundred-yearold phages (if preserved) could still infect hosts that have remained active for the same time, however and to my knowledge, has not been studied. 
Now, consider the possibility that a phage gets aerosolized and is carried in the atmosphere to a very distant location. Is it within reason to assume that it is impossible for this phage to infect a bacterium at its landing site? Probably not, although the chances may be low. But when the low chances cumulate due to the sheer number of airborne phages, it may become a relevant factor. It appears possible that regardless of the host-parasite arms race, phages of distant origin may sometimes launch an infection in a host that it has not seen in a while - this is the take home message of the first century of phage isolation attempts (Örmälä and Jalasvuori, 2013). On the other hand, it is possible that atmosphere itself reduces the viability of viruses via radiation, temperature or dryness and hence extensive airtime (and thus longer flight distance) would also reduce viability remarkably. But if the conditions in the atmosphere eliminates only a fraction of phages, then infective viruses from various different locations may still be raining down from the sky. What could this mean for local evolutionary trends among bacteria? It is an open question, but it is possible that phage migration can potentially influence bacterial evolution and perhaps even be important to phage survival (Morgan et al., 2005).

\section{Rain of genetic elements}

At least some of the airborne bacteria are viable (for review, see Fröhlich-Nowoisky et al., 2016). Cultivable bacteria are present in rain water and in higher atmosphere, therefore even if aerosolized viruses were all dead, their hosts are not. Some, such as Pseudomonas syringae, can modulate their own descend by facilitating the formation of ice nuclei from the atmospheric precipitation (Morris et al., 2010). Various plant pathogens, including bacteria, are known to successfully change habitats across the atmosphere (Morris and Sands, 2017). However, bacteria which are not adapted to their new landing site and the community therein are unlikely to able to establish themselves as a part of the local system. Yet, just by landing to the area bacteria are providing their genetic material to be absorbed by the local community. Naked DNA can be acquired by competent bacteria and assimilated into their genomes (Mell and Redfield, 2014). These genetic transformants (recombinants) are subjected to selection/competition alongside with the contemporaries lacking the distantly originating DNA. However, even before the recently landed bacteria face the potential demise, it can actively disseminate its mobile genetic elements to the locally adapted microbiome (Klumber et al., 2015). Conjugative plasmids, integrative and conjugative elements, activated prophages - and within these elements various types of transposons, insertion sequences and hitchhiking genes - may penetrate the bacterial cell boundaries and thus provide novel genetic material (and new parasites) to the community (Jalasvuori and Koonin, 2015). We may ask whether foreign genetic fragments and elements have any global or local differences in a world that is nevertheless immersed in microbes? I proceed to discuss this possibility. 


\section{Should bacteria remain genetically compatible with their distant relatives?}

One of the existing puzzles in evolutionary biology is the genetic uniformity of organisms across the tree of life. If life has evolved so many ways to defend against (viral) parasites, then why has it not evolved a new genetic code, which would make the host immune to viruses? (Maybe it has, but our current tools designed for conventional organisms are just not suited for finding them.) Naturally, changing the underlying code is not an easy feat once a system has been established (Koonin and Novozhilow, 2009), and if any organism with its complete genome would be able to do that, then it is likely that there is enough time for a virus to chase down the Red Queen (and in essence do the same). Nevertheless, given the same genetic code, genes from archaea can be transferred to bacteria, and they produce the same protein as they did in the organism from which they diverged almost four billion years ago.

Among many possible explanations, the common genetic code has been argued to have emerged due to general optimization for maintaining important codons and for providing the base for innovation-sharing in primordial living systems (Vetsigian et al., 2006). This occurs in computational simulations where, and only where, horizontal gene transfer occurs between simple genetic entities (Sengupta et al., 2014; Aggarwal et al., 2016). Innovation-sharing hypothesis states that it was generally more favorable for early life-forms to be genetically compatible rather than incompatible with other entities, because this allowed them to adopt innovations that emerged in other cell-lines (or even before reliable cell division and the onset of independent cells) (Vetsigian et al., 2006; Jalasvuori et al., 2010).

If compatibility was so exclusively important in early life that only one genetic system emerged, could it be a notable factor in maintaining genetic similarity also today? Indeed, horizontal gene transfer have been observed to have occurred across phylogenetically very distant organisms (Fuchsman et al., 2017). Such adoption of distant genes would be impossible without the uniformity of the underlying genetic alphabet (Koonin and Novozhilov, 2017). Yet, horizontal transfer occurs more frequently, the more closely related the organisms are, although ecological proximity is also an important factor (Kloesges et al., 2011; Smillie et al., 2011). Emergence of deleterious epistatic interactions are more likely when genetic material from long ago diverged organisms meet within a single cell: this is ultimately why speciation occurs (i.e. reproductive barrier develops) in sexually reproducing eukaryotes as chromosomes of individuals are incompatible (Puurtinen et al., 2016).

Would genetic compatibility deteriorate due to mutations and genetic drift more rapidly and possibly eventually to non-existence, if there were no incoming genetic innovations for bacteria to be adopted, i.e. if there was no atmosphere? This is naturally a complex 'what if' question, but it nevertheless may partly 
determine the rate at which de novo compatibility deteriorating mutations are surviving in environmental bacteria and hence the evolutionary tempo in general.

\section{How would natural bacterial communities without an external inflow of genetic material evolve?}

Clearly there is no definite answer to such a question. Yet, short-term evolutionary experiments have been conducted, and they provide a crude framework for the discussion. The longest (and still on-going) microcosm experiment, the famous Lenski Escherichia coli system, the long-term evolution experiment (LTEE), has now been running for over thirty years and 60000 generations (Blount et al., 2018). It shows an interesting characteristic that may give a clue. In half of the replicates of the LTEE, hypermutator strains have taken over the system (Good et al., 2017; Couce et al., 2017). Hypermutators are derivates of the original seeding strain with a highly elevated mutation rate. In Lenski's experiment, it has taken from around few thousand generations to over 30000 generations for these to become dominative species within their local communities.

In this experiment, there is neither an external source of genetic material (innovations) for $E$. coli to take advantage of nor a mean for genes to transfer between the existing strains. In other words, no genes that would have evolved and adapted to a different environment suddenly make an appearance in the system. Should there exist such inflow, sometimes the adoption of foreign genes might help the bacterium to elevate its relative fitness (and thus the fitness of the other genes in that bacterium) over its genetically closely related contemporaries. However, for this to occur, the genetic environment has to be compatible with the adopted gene.

In the absence of horizontal gene transfer and incoming evolutionary innovations there is little selection for the bacteria to remain genetically similar to and hence more compatible with other genetic environments. If there is an evolutionarily favorable change in any of the cells in the closed community, then it has to have acquired this change within the genetic material that was in itself all along. No selection for outside compatibility, only within-cell compatibility.

Hypermutators accumulate genetic distance from other entities very rapidly compared to the communities where mutators did not evolve (Good et al., 2017). The increase in genetic distance is likely to equal to increasing genetic incompatibility. The process is comparable to speciation in sexually reproducing organisms: new species emerge once the epistatic interference of two populations of the same species becomes such that the flow of genes between them becomes impossible (Corbett-Detig et al., 2013; Puurtinen et al., 2016). In practice, some part of a certain gene product has a significantly deleterious or 
altogether lethal interaction with one of the other gene products in its particular genetic environment. This interaction becomes relevant only when genes get mixed between organisms.

In constantly changing environment (which even Lenski's environment is due to the continuous evolution of individual bacteria in the community), there will always likely to be room for new genetic tools that improve the fitness of the particular individual. Even E. coli in different communities have not came up with all the beneficial mutations that have taken place in their replicate systems. Povolotskaya and Kondrasov have also shown that our biosphere has explored only a fraction of all possible viable genetic sequences (Povolotskaya and Kondrashov, 2010). Mutators are good at coming up with both viable and unviable innovations, but simultaneously their capability to adopt genes from other organisms are likely to deteriorate more rapidly (compare this to allopatric speciation of sexually reproducing organisms). When all the genes in the system consist of innovations developed by a relatively few contemporaries, the selection may support mutators that find maximal fitness with a high number of hitchhiking mutations (Couce et al., 2017). However, in contrast, if the system would continuously have a stream of genes from distant relatives, accumulation of random mutations may be a less fit choice for the genetic environment (in practice, the hosting vehicle, the organism) to prevail. Also, mutations are slow in coming up with considerable de novo innovations. In only one of the LTEE communities the ability to grow on citrate has evolved despite of the fact that citrate as an additional source of energy has stably existed in the nutrient broth throughout the 60000 generations (Blount et al., 2012). Now, if horizontal gene transfer was present, what would the probabilities be for within-cell innovation versus external acquirement of this ability?

In conclusion, I here suggest that the inflow of external genetic material can modify the rate at which de novo compatibility decreasing mutations are selected for and ultimately pervading the community. As a result, the overall pace of bacterial divergence from each other slows down (Figure 1). In this process, microbes carried in the atmosphere can have a notable role. Whether or not this is accurate or anything more than a curiosity, remains to be seen. Yet, it may be of interest to identify processes at different temporal and spatial scales that govern microbial evolution on our planet.

\section{Funding}

This work was supported by the Academy of Finland (grant numbers 252411, 297049), Emil Aaltonen Foundation, and Jane and Aatos Erkko Foundation. 


\section{References}

Aggarwal N, Bandhu AV, Sengupta S. Finite population analysis of the effect of horizontal gene transfer on the origin of an universal and optimal genetic code. Phys Biol. 2016. 13:036007.

Atanasova, N., Roine, E., Oren, A., Bamford, D., \& Oksanen, H. (2012). Global network of specific virus-host interactions in hypersaline environments. Environmental Microbiology, 14(2), 426-440.

Barberán A, Henley J, Fierer $\mathrm{N}$ et al. Structure, inter-annual recurrence, and global-scale connectivity of airborne microbial communities. Sci Total Environ 2014;487:187-95.

Braga L, Soucy S, Amgarten D, da Silva A, Setubal J. 2018. Bacterial Diversification in the Light of the Interactions with Phages: The Genetic Symbionts and Their Role in Ecological Speciation. Front. Ecol. Evol. https://doi.org/10.3389/fevo.2018.00006

A. Betts, C. Gray, M. Zelek, R. C. MacLean, K. C. King. High parasite diversity accelerates host adaptation and diversification. Science 2018: Vol. 360: 907-911

Blount, Z. D., R. E. Lenski, and J. B. Losos. 2018. Contingency and determinism in evolution: replaying life's tape. Science 362: 655

Breitbart M, Bonnain C, Malki K, Sawaya NA. Phage puppet masters of the marine microbial realm. Nat Microbiol. 2018. 3:754-766.

Corbett-Detig, R. B. et al. 2013. Genetic incompatibilities are widespread within species. Nature 504: 135137.

Natasha DeLeon-Rodriguez, Terry L. Lathem, Luis M. Rodriguez-R, James M. Barazesh, Bruce E. Anderson, Andreas J. Beyersdorf, Luke D. Ziemba, Michael Bergin, Athanasios Nenes, and Konstantinos T. Konstantinidis. Microbiome of the upper troposphere: Species composition and prevalence, effects of tropical storms, and atmospheric implications. PNAS 2013 110: 2575-2580.

Eduardo Corel,1,^ Philippe Lopez,1 Raphaël Méheust,1 and Eric Bapteste1. Network-Thinking: Graphs to Analyze Microbial Complexity and Evolution. Trends Microbiol. 2016. 24: 224-237.

Juan A. Bonachela, Meike T. Wortel \& Nils Chr. Stenseth. Eco-evolutionary Red Queen dynamics regulate biodiversity in a metabolite-driven microbial system. Scientific Reports 7: 17655 (2017)

Ghoul M, Mitri S. 2016. The Ecology and Evolution of Microbial Competition. Trends in Microbiol. 24: 833845. 
Good BH, McDonald MJ, Barrick JE, Lenski RE, Desai MM. The dynamics of molecular evolution over 60,000 generations. Nature. 2017. 551:45-50

Hiltunen T, Cairns J, Frickel J, Jalasvuori M, Laakso J, Kaitala V, Künzel S, Karakoc E, and Becks L. (2018) Multi-stressor selection alters eco-evolutionary dynamics in experimental communities. Nature Ecology \& Evolution. 2: 1974-1981.

Flores CO, Meyer JR, Valverde S, Farr L, Weitz JS. Statistical structure of host-phage interactions. Proc Natl Acad Sci U S A. 2011. 108:E288-97.

Fuchsman CA, Collins RE, Rocap G, Brazelton WJ. Effect of the environment on horizontal gene transfer between bacteria and archaea. PeerJ. 2017. 5:e3865.

Elina Laanto, Lotta-Riina Sundberg, and Jaana K. H. Bamford. Phage Specificity of the Freshwater Fish Pathogen Flavobacterium columnare. Appl Environ Microbiol. 2011. 77: 7868-7872.

Laanto E, Hoikkala V, Ravantti J, Sundberg LR. Long-term genomic coevolution of host-parasite interaction in the natural environment. Nat Commun. 2017. 8:111.

Labrie SJ, Samson JE, Moineau S. Bacteriophage resistance mechanisms. Nat Rev Microbiol. 2010. 8:317-27. doi: $10.1038 /$ nrmicro2315

Cairns J, Koskinen K, Penttinen R, Patinen T, Hartikainen A, Jokela R, Ruusulehto L, Viitamäki S, Mattila S, Hiltunen T and Jalasvuori M (2018) Black Queen evolution and trophic interactions determine plasmid survival after the disruption of conjugation network. mSystems. 3: e00104-18.

Brockhurst MA1, Morgan AD, Fenton A, Buckling A. 2007. Experimental coevolution with bacteria and phage. The Pseudomonas fluorescens--Phi2 model system. Infect Genet Evol. 7:547-52.

Fröhlich-Nowoisky, J. et al. Bioaerosols in the Earth system: climate, health, and ecosystem interactions. Atmos. Res. 182, 346-376 (2016).

Morris JJ. 2015. Black Queen evolution: the role of leakiness in structuring microbial communities. Trends Genet 31:475-482. doi:10.1016/j.tig.2015.05.004.

Morris JJ, Lenski RE, Zinser ER. 2012. The black queen hypothesis: evolution of dependencies through adaptive gene loss. mBio 3:e00036-12. doi:10.1128/mBio.00036-12.

McFall-Ngai M, Hadfield MG, Bosch TC, Carey HV, Domazet-Lošo T, Douglas AE, Dubilier N, Eberl G, Fukami T, Gilbert SF, Hentschel U, King N, Kjelleberg S, Knoll AH, Kremer N, Mazmanian SK, Metcalf JL, Nealson K, 
Pierce NE, Rawls JF, Reid A, Ruby EG, Rumpho M, Sanders JG, Tautz D, Wernegreen JJ. Animals in a bacterial world, a new imperative for the life sciences. Proc Natl Acad Sci U S A. 2013. 110:3229-36.

Mell JC, Redfield RJ. Natural competence and the evolution of DNA uptake specificity. J Bacteriol. 2014. 196:1471-83.

Jalasvuori M., Jalasvuori M.P. and Bamford J.K.H. (2010). Dynamics of a Laterally Evolving Community of Ribozyme-like Agents as Studied with a Rule-based Computing System. Orig Life Evol Biosph 40, 319-334.

Jalasvuori M. (2012) Vehicles, replicators, and intercellular movement of genetic information: evolutionary dissection of a bacterial cell. Int J Evol Biol 2012, 874153.

Jalasvuori M, Koonin E. (2015) Classification of Prokaryotic Genetic Replicators: Between Selfishness and Altruism. Annals of the New York Academy of Sciences. 1341:96-105.

Klümper U, Riber L, Dechesne A, et al. Broad host range plasmids can invade an unexpectedly diverse fraction of a soil bacterial community. ISME J. 2015. 9:934-945.

Koonin EV, Novozhilov AS. Origin and evolution of the genetic code: the universal enigma. IUBMB Life. 2009. 61:99-111.

Povolotskaya, I., Kondrashov, F. Sequence space and the ongoing expansion of the protein universe. Nature 465, 922-926 (2010) doi:10.1038/nature09105

Koskella B, Brockhurst MA. Bacteria-phage coevolution as a driver of ecological and evolutionary processes in microbial communities. FEMS Microbiol Rev. 2014, 38:916-31. doi: 10.1111/1574-6976.12072.

Mattila S, Ruotsalainen P, Jalasvuori M. (2015) On-demand isolation of bacteriophages against drugresistant bacteria for personalized phage therapy. Frontiers in Microbiology. 6: 1271.

Morgan AD, Gandon S, Buckling A. 2005. The effect of migration on local adaptation in a coevolving hostparasite system. Nature. 437:253-6.

Morris CE, Sands DC, Vanneste JL et al. Inferring the Evolutionary History of the Plant Pathogen Pseudomonas syringae from Its Biogeography in Headwaters of Rivers in North America, Europe, and New Zealand. mBio 2010;1:e00107-10.

O'Brien S, Rodrigues AM, Buckling A. The evolution of bacterial mutation rates under simultaneous selection by interspecific and social parasitism. Proc Biol Sci. 2013. 280:20131913. 
Puurtinen M, Elo M, Jalasvuori M, Kahilainen A, Ketola T, Kotiaho JS, Mönkkönen M, Pentikäinen OT. (2016) Temperature-dependent mutational robustness can explain faster molecular evolution at warm temperatures, affecting speciation rate and global patterns of species diversity. Ecography. 39: 1025-1033.

Sarah Kronheim, Martin Daniel-Ivad, Zhuang Duan, Sungwon Hwang, Andrew I. Wong, lan Mantel, Justin R. Nodwell \& Karen L. Maxwell. A chemical defence against phage infection. Nature 564: 283-286 (2018)

Sengupta S, Aggarwal N, Bandhu AV. Two perspectives on the origin of the standard genetic code. Orig Life Evol Biosph. 2014. 44:287-91.

Smillie CS, Smith MB, Friedman J, Cordero OX, David LA, Alm EJ. Ecology drives a global network of gene exchange connecting the human microbiome. Nature. 2011. 480:241-4. doi: 10.1038/nature10571.

Stilianos Louca et al, Bacterial diversification through geological time, Nature Ecology \& Evolution (2018). DOI: 10.1038/s41559-018-0625-0

Isabel Reche, Gaetano D’Orta, Natalie Mladenov, Danielle M. Winget \& Curtis A. Suttle. Deposition rates of viruses and bacteria above the atmospheric boundary layer. The ISME Journal 12: 1154-1162 (2018)

Arite Wolf, Jutta Wiese, Günter Jost, Karl-Paul Witzel. Wide Geographic Distribution of Bacteriophages That Lyse the Same Indigenous Freshwater Isolate (Sphingomonas sp. Strain B18). Appl. Environ. Microbiol. 69: 2395-2398

Örmälä A-M., Jalasvuori M. (2013) Phage therapy: should bacterial resistance to phages be a concern, even in the long run? Bacteriophage, 3:e24219.

Rodriguez-Valera, F., Martin-Cuadrado, A.-B., Rodriguez-Brito, B., Pasić, L., Thingstad, T. F., Rohwer, F., et al. (2009). Explaining microbial population genomics through phage predation. Nat. Rev. Microbiol. 7, 828836.

Vetsigian K, Woese C, Goldenfeld N. Collective evolution and the genetic code. Proc Natl Acad Sci U S A. 2006. 103(28):10696-701.

Koonin EV, Novozhilov AS. Origin and Evolution of the Universal Genetic Code. Annu Rev Genet. 2017. 51:45-62.

Kloesges T, Popa O, Martin W, Dagan T. Networks of gene sharing among 329 proteobacterial genomes reveal differences in lateral gene transfer frequency at different phylogenetic depths. Mol Biol Evol. 2011. 28:1057-74. 
Alejandro Couce, Larissa Viraphong Caudwell, Christoph Feinauer, Thomas Hindré, Jean-Paul Feugeas, Martin Weigt, Richard E. Lenski, Dominique Schneider, Olivier Tenaillon. Mutator genomes decay during adaptive evolution. Proceedings of the National Academy of Sciences. 2017, 114: E9026-E9035.

Blount ZD, Barrick JE, Davidson CJ, Lenski RE. Genomic analysis of a key innovation in an experimental Escherichia coli population. Nature. 2012 489: 513-518.

Womack AM, Bohannan BJM, Green JL. Biodiversity and biogeography of the atmosphere. Philosophical Transactions of the Royal Society of London B: Biological Sciences 2010;365:3645-53. 


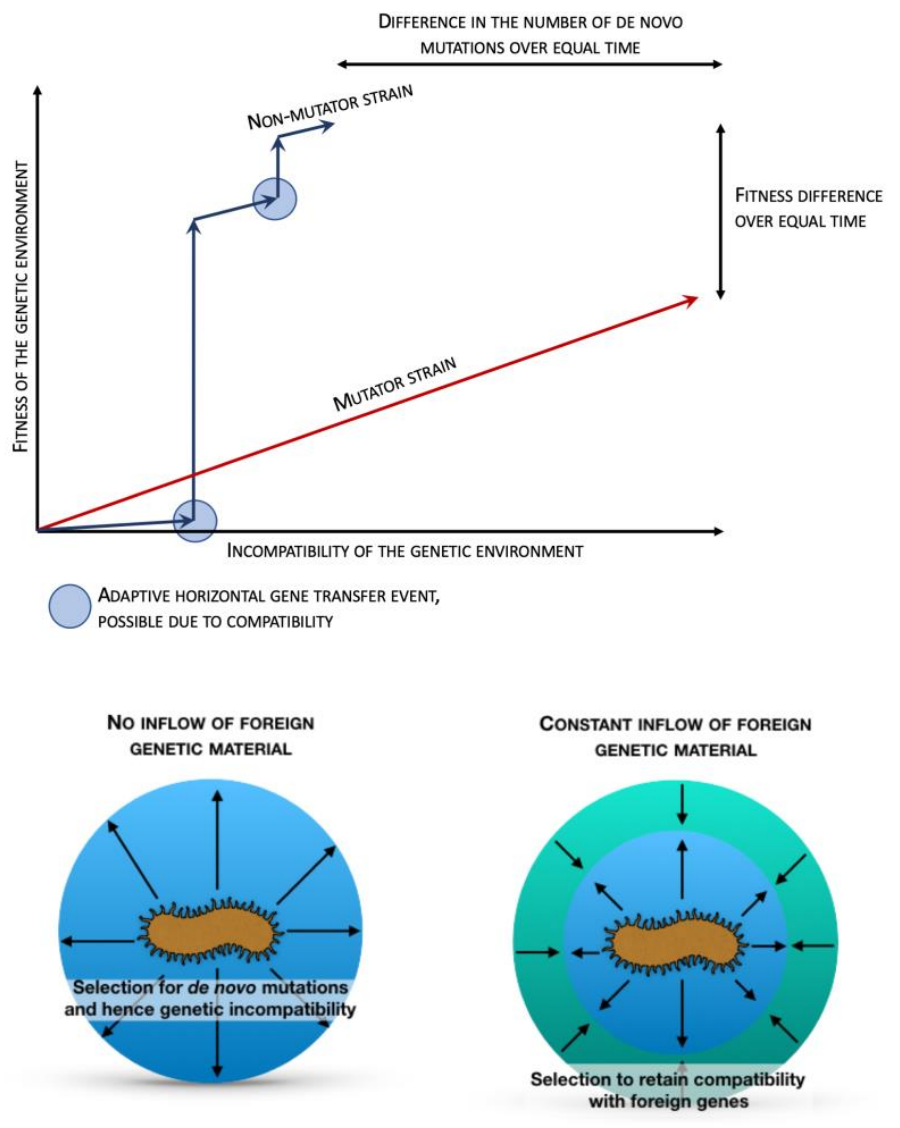

Figure 1. Atmosphere mediates the transfer of microbes across distant habitats. This provides the established bacterial communities with new genetic material that may elevate the fitness of the recipient, but only if the material is compatible with the prevailing genetic environment. This can select for compatible genomes, and hence, compared to systems without the transfer, slow down the pace at which mutations accumulate in genomes. 